Published in final edited form as: Acta Obstet Gynecol Scand. 2011 December ; 90(12): 1379-1385. doi:10.1111/j. 1600-0412.2011.01275.x.

\title{
Epidemiology of stillbirth in low-middle income countries: A Global Network Study
}

\author{
ELIZABETH M. McCLURE ${ }^{1}$, OMRANA PASHA ${ }^{2}$, SHIVAPRASAD S. GOUDAR ${ }^{3}$, ELWYN \\ CHOMBA $^{4}$, ANA GARCES ${ }^{5}$, ANTOINETTE TSHEFU ${ }^{6}$, FERNANDO ALTHABE ${ }^{7}$, FABIAN \\ ESAMAI $^{8}$, ARCHANA PATEL $^{9}$, LINDA L. WRIGHT ${ }^{10}$, JANET MOORE ${ }^{1}$, BHALCHANDRA S. \\ KODKANY $^{3}$, JOSE M. BELIZAN ${ }^{7}$, SARAH SALEEM ${ }^{2}$, RICHARD J. DERMAN ${ }^{11}$, WALDEMAR \\ A. CARLO ${ }^{12}$, K. MICHAEL HAMBIDGE ${ }^{13}$, PIERRE BUEKENS ${ }^{14}$, EDWARD A. LIECHTY ${ }^{15}$, \\ CARL BOSE ${ }^{16}$, MARION KOSO-THOMAS ${ }^{2}$, ALAN H. JOBE ${ }^{17}$, ROBERT L. GOLDENBERG ${ }^{18}$, \\ and the Global Network Investigators \\ ${ }^{1}$ Research Triangle Institute, Durham, NC, USA \\ ${ }^{2}$ Aga Khan University, Karachi, Pakistan \\ ${ }^{3}$ Jawaharlal Nehru Medical College, Belgaum, India \\ ${ }^{4}$ University of Zambia, Lusaka, Zambia \\ ${ }^{5}$ San Carlos University, Guatemala City, Guatemala \\ ${ }^{6}$ Kinshasa School of Public Health, Kinshasa, DRC \\ ${ }^{7}$ University of Buenos Aires, Buenos Aires, Argentina \\ ${ }^{8}$ Moi University, Eldoret, Kenya \\ 9 Indira Medical College, Nagpur, India \\ ${ }^{10}$ Eunice Kennedy Shriver National Institute of Child Health and Human Development, Bethesda, \\ MD, USA \\ ${ }^{11}$ Christiana Health Care, Newark, DE, USA \\ ${ }^{12}$ University of Alabama at Birmingham, Birmingham, AL, USA \\ ${ }^{13}$ University of Colorado Health Sciences Center, Denver, CO, USA \\ ${ }^{14}$ Tulane School of Public Health and Tropic Medicine, New Orleans, LA, USA \\ ${ }^{15}$ Indiana University, Indianapolis, IN, USA \\ ${ }^{16}$ University of North Carolina at Chapel Hill, Chapel Hill, NC, USA \\ ${ }^{17}$ University of Cincinnati, Cincinnati, $\mathrm{OH}$ \\ ${ }^{18}$ Drexel University, Philadelphia, PA, USA
}

\begin{abstract}
Objective-To determine population-based stillbirth rates and to determine whether the timing and maturity of the stillbirths suggest a high proportion of potentially preventable deaths.

(C) 2011 Nordic Federation of Societies of Obstetrics and Gynecology

Correspondence Elizabeth McClure, Research Triangle Institute, Durham, NC, USA. mcclure@ rti.org.

Conflict of interest

The authors have stated explicitly that there are no conflicts of interest in connection with this article.
\end{abstract}


Design-Prospective observational study.

Setting-Communities in six low-income countries (Democratic Republic of Congo, Kenya, Zambia, Guatemala, India, and Pakistan) and one site in a mid-income country (Argentina).

Population-Pregnant women residing in the study communities.

Methods-Over a five-year period, in selected catchment areas, using multiple methodologies, trained study staff obtained pregnancy outcomes on each delivery in their area.

Main outcome measures-Pregnancy outcome, stillbirth characteristics.

Results-Outcomes of 195400 deliveries were included. Stillbirth rates ranged from 32 per 1 000 in Pakistan to 8 per 1000 births in Argentina. Three-fourths (76\%) of stillbirth off-spring were not macerated, $63 \%$ were 237 weeks and $48 \%$ weighed $2500 \mathrm{~g}$ or more. Across all sites, women with no education, of high and low parity, of older age, and without access to antenatal care were at significantly greater risk for stillbirth $(p<0.001)$. Compared to those delivered by a physician, women delivered by nurses and traditional birth attendants had a lower risk of stillbirth.

Conclusions-In these low-middle income countries, most stillbirth offspring were not macerated, were reported as $\geq 37$ weeks' gestation, and almost half weighed at least $2500 \mathrm{~g}$. With access to better medical care, especially in the intrapartum period, many of these stillbirths could likely be prevented.

\section{Keywords}

Developing countries; intrapartum stillbirth; stillbirth

\section{Introduction}

An estimated 3.2 million stillbirths occur worldwide each year with more than $98 \%$ of these in low to middle-income countries $(1,2)$. Stillbirth rates may be as high as 30-40 per 1000 births in low-income countries, especially areas of sub-Saharan Africa and south Asia, while rates as low as 3-4 per 1000 are reported in many high-income countries (2-8). In lowincome countries, a gestational age of 28 weeks or a birth-weight of $1000 \mathrm{~g}$ is often selected as the lower limit of viability (4). In high-income countries, stillbirth has generally been defined as a fetal loss beyond 20 or 22 weeks; however, some high-income countries such as Sweden have used 28 weeks as the lower cutoff as recently as 2010 (9). The World Health Organization has recommended using a gestational age and birthweight cutoff of 28 weeks and $1000 \mathrm{~g}$, respectively, as the lower limits for international comparisons (4). Health care providers in high-income countries frequently determine whether the stillbirth occurred in the intrapartum period. When the timing of the fetal death is not available, the extent of skin maceration is often used as a surrogate measure. Stillbirths that occur more than 12-24 hours prior to delivery generally have skin that is macerated, whereas those occurring in the intrapartum period or immediately prior to or during delivery generally have intact skin (10). In high-income countries, fewer than $10 \%$ of all stillbirths are intrapartum, whereas in some countries with minimal resources, up to half of all stillbirths are thought to occur in the intrapartum period $(3,10)$. Intrapartum stillbirths, especially those occurring close to term, are generally considered potentially preventable, as they often are often associated with limited access to, or poor quality of, obstetric care $(2,10)$.

Because stillbirths are not reported routinely in the vital statistics in many low-income countries, much is still unknown about the timing and circumstances associated with these stillbirths, where about half of all deliveries occur at home $(11,12)$. For many reasons, stillbirth has been under-studied, under-reported, and rarely considered in attempts to 
improve pregnancy outcomes in low-income countries. We sought to determine populationbased stillbirth rates and to characterize health care at delivery in prospective, well defined community-based birth cohorts in community settings in low-middle income countries.

\section{Material and methods}

The study was conducted by the Global Network for Women's and Children's Health Research (Global Network), a National Institutes of Health-funded, multi-country research network. The study was conducted in nine sites in seven countries: Argentina, Guatemala, Democratic Republic of Congo (DRC), Kenya and Zambia, India (sites in Orissa, Belgaum, Nagpur), and Pakistan. Prospective data registries were initially created to establish baseline delivery and pregnancy outcome rates for the FIRST BREATH Trial, a trial of neonatal resuscitation training to improve birth outcomes (13-15). The registries were then maintained and expanded to additional sites and included a total of 195400 deliveries and 4 301 stillbirths at the end of the study period. This study was reviewed and approved by the institutional ethics review committees of all participating study sites, the partner institutions in the USA and the data center, Research Triangle Institute. Consent was obtained at the community level; women provided informed consent to collect data regarding their pregnancy and its outcome.

Each Global Network site included six to 28 distinct geographic clusters, generally representing health districts, with approximately 300-500 annual births per cluster. The outcomes of all deliveries occurring in the clusters were collected. Each site went to great lengths to ensure completeness of pregnancy outcome data for all births. Data collection was overseen by trained study coordinators (health workers, nurses or physicians). Each cluster had one or more paid registry administrators to collect data. Community birth attendants were also provided with clean delivery kits with the expectation that they would notify the study team of each delivery, and in any case were contacted by study staff on a regular basis to ascertain any recent deliveries. Data were monitored on a monthly basis to make sure that there were no unexplained inconsistencies over time. In sites where some or all women delivered in hospitals, hospital logs were routinely checked for women living in the study clusters. Sites also utilized additional culturally appropriate methods to ensure completeness of reporting. In one country, for example, village elders were provided with a cell phone to notify the study team of deliveries in his village. In addition, as another effort to identify all deliveries, pregnant women were generally identified and registered between 24 weeks of pregnancy and delivery. Finally, in those areas which had vital records available, these were periodically reviewed for cases missed by our other types of surveillance. We believe that by using multiple methods of ascertainment, we came very close to achieving $100 \%$ identification of all pregnancies and delivery outcomes.

Active birth attendants were prospectively trained to collect data and assess basic clinical variables and outcomes, including differentiation of stillbirths and neonatal deaths at birth, type of stillbirth, birthweight, and assessment of gestational age. Birth attendants were also taught to distinguish 'macerated' from 'fresh' stillbirths using pictures depicting forms of maceration. After delivery, the study coordinator collected the data recorded by the birth attendant. Data included maternal demographics, and neonatal and maternal outcomes at delivery. A stillbirth was defined as any delivery greater than or equal to $1000 \mathrm{~g}$, corresponding to approximately 28 weeks' gestation, in which no signs of life (breathing, crying, heartbeat, movement) were evident. Stillbirth offspring were identified as having signs of maceration or not. Gestational age was estimated using last menstrual period (LMP), or clinical data such as physical examination, ultrasound and other available information when LMP was unknown. The birthweight was taken within 48 hours of delivery using study scales. When birthweight could not be obtained by scale, weight was 
estimated to distinguish infants weighing less than and greater than $1000 \mathrm{~g}$ and $2500 \mathrm{~g}$. Models and pictures were used to teach these concepts to community birth attendants. The type of delivery attendant included physician, nurse or equivalent, traditional birth attendant (TBA), family or unattended. Location of delivery included hospital, health center or home (including the TBA's home or in transit). Finally, antenatal care was defined as having at least one visit with a skilled health provider.

All data were entered at each study site and transmitted for central analyses; data edits, including inter- and intra-form consistency checks, were performed at entry with additional edits performed by the data center. The relationships between stillbirth prevalence and key demographic and clinical characteristics (maternal age and education, parity, antenatal care visits, type of birth attendant, and delivery location) were evaluated using log binomial multivariate regression models with generalized estimating equations (GEE) used to account for correlation of events within clusters. $p$-values were calculated for both single and multivariate analyses. The data were analyzed using sas-version 9.2 (Cary, NC, USA).

\section{Results}

From March 2005 to August 2010, of the 198436 pregnant women identified, the study recorded 195400 births which were $\geq 1000 \mathrm{~g}$ in the 129 study clusters. A total of 4301 stillbirths were recorded and the mean $\geq 1000 \mathrm{~g}$ stillbirth rate across all births in all sites was 22 per 1000 deliveries, ranging from 8 per 1000 in Argentina to 32 per 1000 deliveries in Pakistan (Table 1). About three-quarters (76.4\%) of the stillbirth offspring had intact skin with no signs of maceration, with a range of 56.9-87.7\% among the sites. Birthweights were available for $83 \%$ of stillbirths and $99 \%$ overall. The mean birth weight for the stillbirth offspring for which we had data was $2423 \mathrm{~g}$, with a range of mean birthweights among the sites of $2199-3004 \mathrm{~g}$. For all births, the mean birthweight was $2883 \mathrm{~g}$, with a range of 2 598-3 008g among the sites (data not shown). Of the stillbirths, $47.9 \%$ weighed $\geq 2500 \mathrm{~g}$. The majority of stillbirths (63.4\%) were recorded as 237 weeks (or term) and overall, $95.6 \%$ of the births were recorded as $\geq 37$ weeks.

Most women (93.5\%) received at least one antenatal care visit, with rates ranging from $85.6 \%$ in Kenya to $99.9 \%$ in Nagpur, India (data not shown). In Argentina, $99 \%$ of the deliveries were conducted by a skilled health provider (physician or nurse), whereas in five sites (DRC, Kenya, Zambia, Pakistan, and Guatemala), fewer than 50\% of deliveries were conducted by a skilled health provider (Figure 1). In those sites, fewer than $5 \%$ of the deliveries were conducted by a physician. Fewer than half (42.8\%) of the deliveries were conducted in a health care facility (hospital or health center). Cesarean section rates ranged from less than $1 \%$ in the DRC and Orissa, India to $22.1 \%$ in Argentina. Five of the remaining sites had Cesarean section rates of $3 \%$ or less.

Table 2 presents characteristics among women with and without stillbirths and relative risks for stillbirth. Women who were older than 35 years of age at delivery, those who had no formal education, and those who received no prenatal care had a significantly higher risk of stillbirth compared to those without these risk factors. Women who were primiparous or who were in their $5^{\text {th }}$ or more pregnancy also had increased risk of stillbirth compared to women in their second through fourth pregnancies. There were also significant differences in delivery outcomes by the type of birth attendant. The risk of stillbirth was higher among physician deliveries than among deliveries by nurses and traditional birth attendants. Stillbirth rates were generally similar across delivery locations and, when adjusted for other variables, the birth location was not a significant risk factor for stillbirth. 


\section{Discussion}

We captured delivery outcomes for almost 200000 births in distinct, geographically defined clusters in seven countries, representing one of the largest studies of this kind to date. Although other studies have reported hospital-based stillbirth rates, few to date have examined hospital and community births to estimate the overall population rates for defined geographic areas $(6,7,15)$. The overall stillbirth rate of 22 per 1000 births, with individual country rates ranging from 8 per 1000 in the Argentinean clusters to 32 per 1000 in the Pakistani clusters, closely approximates the estimated country rates reported for these countries in some modeling studies $(1,2)$. These rates are significantly greater than the rates of three to five per 1000 reported from many high-income countries.

Stillbirth offspring weighing $<1000 \mathrm{~g}$, or those occurring at about 20-28 weeks, were not included in this study but are often included in reported USA and other high-income country rates. In the USA, for example, stillbirths occurring at 20-27 weeks account for almost half of the reported stillbirths and similar results have been reported in other high income countries $(16,17)$. Thus, the disparity between the stillbirth rates between high and low income countries may be even larger than suggested by our results.

When examining the socio-demographic and health care characteristics, the risk factors for stillbirth were consistent with previous studies from both low and high income countries (7,17-20). Women $>35$ years of age, those who had no formal schooling, who received no prenatal care, and who were either primiparous or with grand-multiparity ( $\geq 5$ prior pregnancies) were also at greater risk to experience a stillbirth.

Access to health care was of particular interest. For the entire study sample, $94 \%$ of the women had at least one antenatal care visit, $51 \%$ delivered at home, $16 \%$ had no health care provider available at delivery, and $34 \%$ had an unskilled attendant. Only $4 \%$ had a Cesarean delivery, with rates of less than $1 \%$ reported in several countries. Women who had no antenatal care had a higher risk for a stillbirth compared to those women with antenatal care. Although we did not formally test the association between stillbirth and Cesarean section due to the confounding factors reported elsewhere (21-24), with one exception, the sites with the lowest Cesarean section rates tended to have the highest stillbirth rates. Of interest, in Nagpur, India, $17 \%$ of the births were delivered by Cesarean section, but the stillbirth rate was high (22 per 1000 births). Rising Cesarean rates in low-resources settings are a topic of recent debate, especially as the Cesareans in these settings may be done for the wrong indications or may not improve outcome (23). However, when performed on the appropriate patients, it remains clear that Cesarean section can save maternal and fetal lives and that a Cesarean section rate of at least $5 \%$ deliveries represents the minimal rate necessary to address preventable perinatal mortality, as recommended by a number of international organizations (24).

The observation that among the providers, the physicians had the highest stillbirth rates, and that stillbirth rates did not differ markedly whether the delivery occurred in a home clinic or hospital, led us to speculate about the potential reasons for these observations. Most likely, women with high risk conditions such as bleeding or seizures may have been more likely to seek care with physicians in facilities. However, many facilities at our sites are characterized by limited equipment, few medicines, and long waits for life-saving interventions such as Cesarean section. Whether a physician was present or not, the lack of resources might explain the failure of facility or physician delivery to substantially reduce stillbirth rates.

This study had several strengths. First, it is one of the largest prospective studies to register women during pregnancy and to capture birth outcomes for a representative populationbased sample. Because the study was conducted in geographic areas where a substantial 
proportion of the births occurred at home, data from this study are likely to be more representative of these geographic areas than many hospital-based studies. In addition, we used trained coordinators to supervise and evaluate the quality of the data collected, as well as a number of methods to ensure that data from all births were captured. Few low-income country studies of stillbirth in communities have captured birthweight or gestational age, often because of cultural or other barriers (1). By repeated training, we worked very hard with our study staff to identify barriers to registering stillbirthsand, where applicable,to overcome their initial reluctance to handle or register stillbirth offspring.

One of the potential limitations of the study that may have resulted in reporting bias was our inability to ensure that all births were captured; however, in each site, our staff used several methods to determine who in each community was pregnant and who had delivered. In addition, the pregnancy outcomes obtained by our staff were compared with the available health records. Finally, the evaluation of delivery and stillbirth rates and inter-cluster variability ensured that large unexplained variations in either the birth or stillbirth rates were not occurring over time. Another potential limitation is the quality of the data collected for unattended births. To address this issue, our study coordinators interviewed each mother and other family members. Obtaining information on these home births is critical as they represent a substantial proportion of the live births and of the stillbirths in these geographic areas.

Still another potential limitation deals with the data regarding maceration. Despite training on this issue using graphic pictures and descriptions, it is possible that mild cases of maceration were misclassified. However, our results are in the same direction as other studies which have found that fewer than half of all stillbirths in low-income countries are macerated $(2,10)$. Signs of maceration have been used as one indicator to distinguish antepartum from intrapartum stillbirth (10,20). However, we expect that an unknown proportion of the non-macerated stillbirths occurred prior to labor, and recognize that the concordance between 'non-maceration' and intrapartum death is not $100 \%$. Finally, a potential limitation to the data relates to the estimates of gestational age. Because few of the sites had ultrasound to confirm LMP-derived gestational ages, we suspect that the actual percent of term births and especially term stillbirths may have been somewhat lower than the numbers reported here. Nevertheless, because the gestational age and birth-weight distributions paint a similar picture, we are confident that most stillborn offspring were born at or near term, and most were not macerated.

A primary gap in stillbirth research and policy planning has been the reliance on hospital studies to estimate stillbirth rates, especially as the majority of the stillbirths worldwide occur in home settings and are never registered, and thus no vital statistics are available $(11,25)$. The mean stillbirth rate of more than 22 per 1000 births represents more than a five-fold increase compared to high-income country rates (1). An important observation is that in the less developed communities, where most deliveries occurred in home settings without trained health providers, rates were as high as 32 per 1000 , compared to the stillbirth rates in Argentina of 8 per 1000 , where nearly all deliveries occurred in hospital settings. Of particular note, the majority of the stillbirths occurred at term and had no signs of maceration and almost half were $\geq 2500 \mathrm{~g}$. Thus, if delivered before fetal death, most infants should not suffer the adverse effects of preterm birth and would likely survive. These results strongly suggest that the majority of stillbirths could be salvaged with access to appropriate obstetrical care. Although our data suggest that higher quality of health care at delivery, especially access to health care providers and appropriate Cesarean section, is associated with lower stillbirth rates, more research on the specific causes of these stillbirths would assist in defining appropriate interventions. Our findings suggest that the majority of stillbirths in these developing countries could be prevented. 


\section{Acknowledgments}

Global Network investigators: Argentina - Fernando Althabe and Jose M. Belizan, (Institute for Clinical Effectiveness and Health Policy); Democratic Republic of Congo - John Ditekemena and Antoinette Tshefu (Kinshasa School of Public Health); Guatemala - Ana Garces (San Carlos University); India - Arjit Mohapatra and Sailajanandan Parida (Sriramchandra Bhanja Medical College), Shivaprasad S. Goudar and BhalchandraS.Kodkany(JawaharlalNehruMedicalCollege); Kenya - Fabian Esamai, Hillary Mabaye, Constance Tenge (Moi University); Pakistan - Sarah Saleem, Neelofar Fariq, and Omrana Pasha (Aga Khan University); Zambia - Albert Manasyan and Elwyn Chomba (University of Zambia); USA - Waldemar A. Carlo (University of Alabama at Birmingham); Nancy Krebs and Michael Hambidge (University of Colorado), Robert L. Goldenberg (Drexel University); Pinaki Panigrahi (University of Maryland Baltimore), Richard J. Derman (University of Missouri, Kansas City), Carl Bose (University of North Carolina at Chapel Hill), Pierre Buekens (Tulane School of Public Health and Tropical Medicine), Patricia Hibberd (Massachusetts General), Edward A Liechty (Indiana University), Linda L. Wright and Marion Koso-Thomas (Eunice Kennedy Shriver National Institute of Child Health and Human Development), Janet Moore, Elizabeth M. McClure, Tracey Grant, and Dennis Wallace (Research Triangle Institute).

Funding

Study supported by NIH grants from the Global Network for Women's and Children's Health Research (U01 HD040477, U01, HD0434475, U01 HD043464, U01 HD040657, U01 HD042372, U01 HD040607, U01 HD040636).

\section{References}

1. Stanton C, Lawn JE, Rahman H, Wilczynska-Ketende K, Hill K. Stillbirth rates: delivering estimates in 190 countries. Lancet. 2006; 367:1487-94. [PubMed: 16679161]

2. Lawn J, Shibuya K, Stein C. No cry at birth: global estimates of intrapartum stillbirth and intrapartum-related neonatal deaths. Bull World Health Org. 2005; 83:409-17. [PubMed: 15976891]

3. McClure EM, Saleem S, Pasha O, Goldenberg RL. Stillbirth in developing countries: a review of causes, risk factors and prevention strategies. J Matern Fetal Neonatal Med. 2009; 22:183-90. [PubMed: 19089779]

4. World Health Organization. World Health Report 2005: Make every mother and baby count. WHO; Geneva: 2005.

5. Burke L, Suswardany DL, Michener K, Mazurki S, Adair T, Elmiyati C, Rao C. Utility of local health registers in measuring perinatal mortality: a case study in rural Indonesia. BMC Pregnancy Childbirth. 2011; 11:20. [PubMed: 21410993]

6. Fawole AO, Shah A, Tongo O, Dara K, El-Ladan AM, Umezulike AC, et al. Determinants of perinatal mortality in Nigeria. Int J Gynaecol Obstet. 2011; 114:37-42. [PubMed: 21489535]

7. Cousens S, Blencowe H, Stanton C, Chou D, Ahmed S, Steinhardt L, et al. National, regional, and worldwide estimates of stillbirth rates in 2009 with trends since 1995: a systematic analysis. Lancet. 2011; 377:1319-30. [PubMed: 21496917]

8. McClure EM, Goldenberg RL, Bann CM. Maternal mortality, stillbirth and measures of obstetric care in developing and developed countries. Int J Gynaecol Obstet. 2007; 96:139-46. [PubMed: 17274999]

9. Fellman J, Eriksson AW. Stillbirth rates in singletons, twins and triplets in Sweden, 1869 to 2001. Twin Res Hum Genet. 2006; 9:260-5. [PubMed: 16611496]

10. Goldenberg RL, McClure EM, Bann CM. The relationship of intrapartum and antepartum stillbirth rates to measures of obstetric care in developed and developing countries. Acta Obstet Gynecol Scand. 2007; 86:1303-9. [PubMed: 17963057]

11. Darmstadt GL, Lee AC, Cousens S, Sibley L, Bhutta ZA, Donnay F, et al. 60 million non-facility births: who can deliver in community settings to reduce intrapartum-related deaths? Int J Gynaecol Obstet. 2009; 107:S89-112. [PubMed: 19815200]

12. Goldenberg RL, McClure EM, Belizán JM. Commentary: reducing the world's stillbirths. BMC Pregnancy Childbirth. 2009; 9(Suppl 1):S1. [PubMed: 19426464] 
13. Carlo WA, Goudar SS, Jehan I, Chomba E, Tshefu A, Garces A, et al. First Breath Study Group. Newborn-care training and perinatal mortality in developing countries. N Engl J Med. 2010; 362:614-23. [PubMed: 20164485]

14. Carlo WA, Goudar SS, Jehan I, Chomba E, Tshefu A, Garces A, et al. First Breath Study Group. High mortality of very-low-birth-weight infants born in communities in developing countries despite birth attendant training. Pediatrics. 2010; 126:e1072-80. [PubMed: 20937655]

15. McClure EM, Wright LL, Goldenberg RL, Goudar SS, Parida SN, Jehan I, et al. NICHD FIRST BREATH Study Group. The global network: a prospective study of stillbirths in developing countries. Am J Obstet Gynecol. 2007; 197:247.e1-5. [PubMed: 17826406]

16. MacDorman MF, Kirmeyer S. Fetal and perinatal mortality, United States, 2005. Natl Vital Stat Rep. 2009; 57:1-19. [PubMed: 19294965]

17. Fretts R. Stillbirth epidemiology, risk factors, and opportunities for stillbirth prevention. Clin Obstet Gynecol. 2010; 53:588-96. [PubMed: 20661043]

18. Kupka R, Kassaye T, Saathoff E, Hertzmark E, Msamanga GI, Fawzi WW. Predictors of stillbirth among HIV-infected Tanzanian women. Acta Obstet Gynecol Scand. 2009; 88:584-92. [PubMed: 19306132]

19. Audu BM, Alhaji MA, Takai UA, Bukar M. Risk factors for stillbirths at university of Maiduguri teaching hospital, Maiduguri, Nigeria: A cross-sectional retrospective analysis. Nig Med J. 2009; 50:42-6.

20. Getahun D, Ananth CV, Kinzler WL. Risk factors for antepartum and intrapartum stillbirth: a population-based study. Am J Obstet Gynecol. 2007; 196:499-507. [PubMed: 17547873]

21. Lumbiganon P, Laopaiboon M, Gülmezoglu AM, Souza JP, Taneepanichskul S, Ruyan P, et al. World Health Organization Global Survey on Maternal and Perinatal Health Research Group. Method of delivery and pregnancy outcomes in Asia: the WHO global survey on maternal and perinatal health 2007-08. Lancet. 2010; 375:490-9. [PubMed: 20071021]

22. Festin MR, Laopaiboon M, Pattanittum P, Ewens MR, Henderson-Smart DJ, Crowther CA, SEAORCHID Study Group. Caesarean section in four South East Asian countries: reasons for, rates, associated care practices and health outcomes. BMC Preg Childbirth. 2009; 9:17.

23. Stanton C, Ronsmans C, Baltimore Group on Cesarean. Recommendations for routine reporting on indications for cesarean delivery in developing countries. Birth. 2008; 35:204-11. [PubMed: 18844646]

24. Yakoob MY, Lawn JE, Darmstadt GL, Bhutta ZA. Stillbirths: epidemiology, evidence, and priorities for action. Semin Perinatol. 2010; 34:387-94. [PubMed: 21094413]

25. Darmstadt GL, Yakoob MY, Haws RA, Menezes EV, Soomro T, Bhutta ZA. Reducing stillbirths: interventions during labour. BMC Pregnancy Childbirth. 2009; 9(Suppl 1):S6. [PubMed: 19426469] 


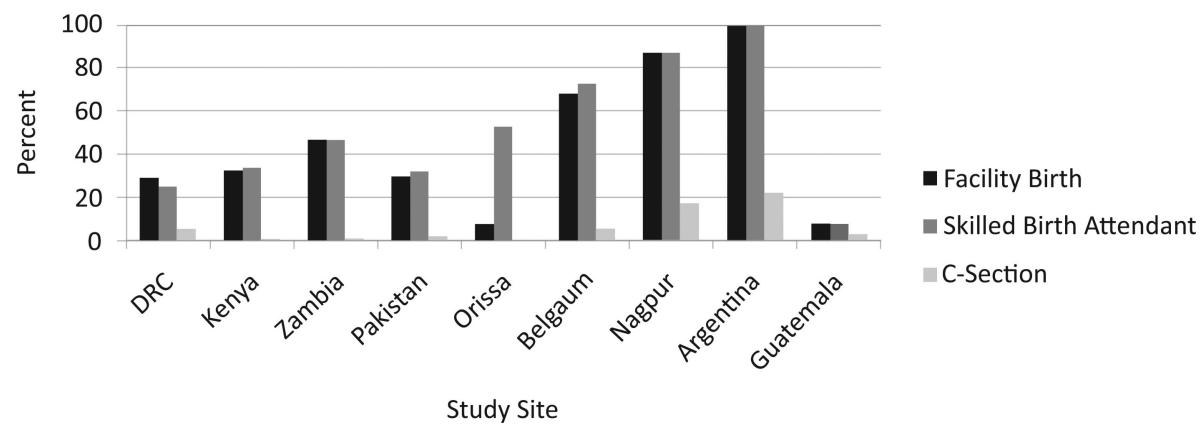

Figure 1.

Health care characteristics at delivery by site. 


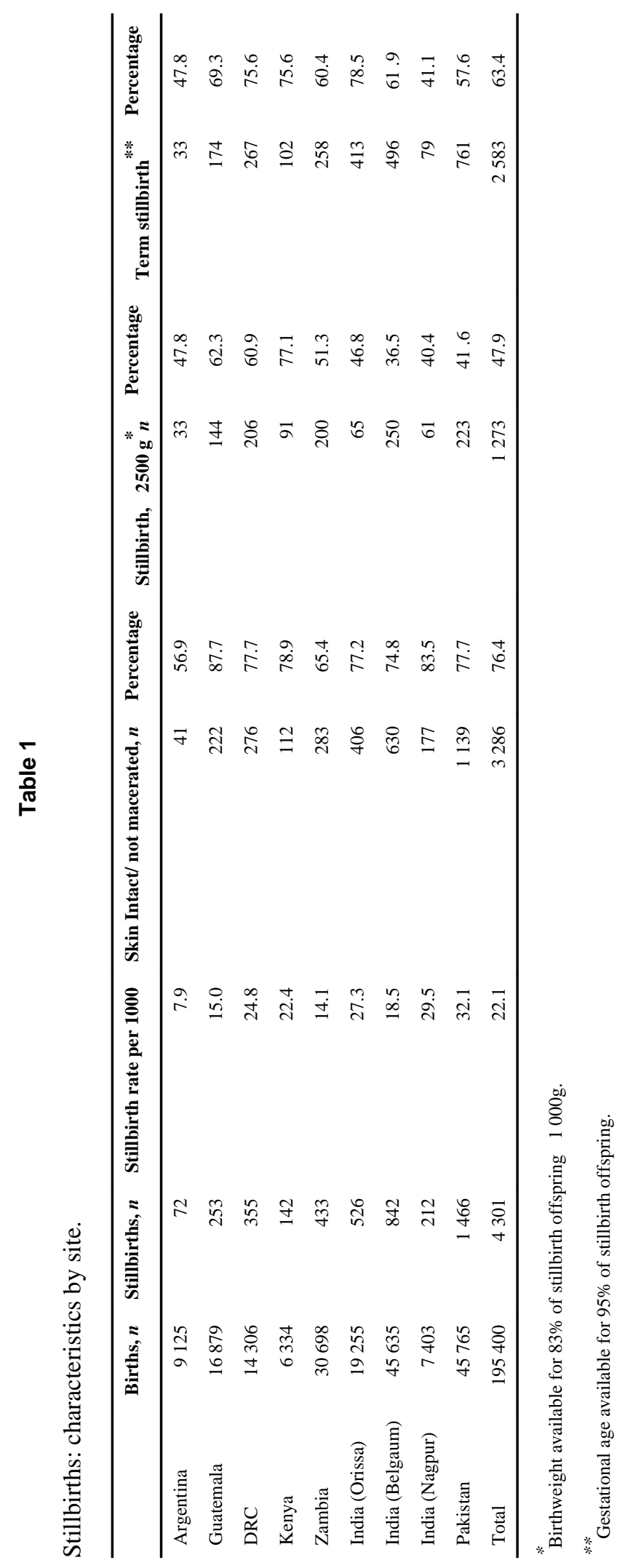


